\title{
Large Mediastinal Mass in Pregnancy: Utility of Echocardiography and Cardiac MRI
}

\author{
Giuseppina Novo ${ }^{\mathrm{a}, \mathrm{d}}$, Antonino Rotolo ${ }^{\mathrm{a}}$, Salvatore Montalto ${ }^{\mathrm{a}}$, Giuseppe Coppola ${ }^{\mathrm{a}}$, Michele Farinella ${ }^{\mathrm{a}}$, \\ Gianfranco Ciaramitaro ${ }^{a}$, Emanuele Grassedonia ${ }^{\mathrm{b}}$, Fabrizia Centineo ${ }^{\mathrm{a}}$, Giovanni Ruvolo ${ }^{\mathrm{a}}$, \\ Salvatore Novo ${ }^{a}$, Pasquale Assennato ${ }^{a}$
}

\begin{abstract}
A 29-year-old woman at the 27 th week of gestation was admitted to the hospital for dyspnea, chest pain radiating to the left shoulder and fatigue. An echocardiogram showed the presence of a pericardial effusion and an inhomogeneously echogenic mass, before the right ventricle, in the mediastinum, that modified the geometry of the right ventricle without impairing its filling. A cardiac MRI, subsequently performed, better defined the mass dimension and its contiguity relationship; moreover, it confirmed the suspicion of a lymphoproliferative disease. A mediastinal biopsy showed an infiltrating non-Hodgkin's lymphoma at B big cells. After waiting until the 30th week of gestation, we proceeded to caesarean delivery, excellently succeeded, and contemporaneously to mass excision. Echocardiography was able to identify the presence of the mediastinal mass and to monitor its consequences on cardiac haemodynamic. Cardiac magnetic resonance was performed safely after the third trimester without producing apparent problems in the newborn. It helped in giving information on the mass dimension and its contiguity relationship but didn't characterize the mass.
\end{abstract}

Keywords: Mass; Lymphoma; Echocardiography; Magnetic resonance

\section{Introduction}

Non-Hodgkin lymphoma represents a rare clinical entity during pregnancy and in literature there are no clear guide-

\footnotetext{
Manuscript accepted for publication November 17, 2010

${ }^{\mathrm{a}}$ Division of Cardiology, Department of Internal Medicine and Cardiovascular Disease, University of Palermo, Italy

${ }^{\mathrm{b}}$ Radiology Department, University of Palermo, Italy

${ }^{\mathrm{c}}$ Department of Cardiac Surgery, University of Palermo, Italy

${ }^{\mathrm{d}}$ Corresponding author: Del Vespro street 127, 90129 Palermo, Italy.

Email: novog@unipa.it

doi: $10.4021 /$ jmc $92 w$
}

lines about its diagnosis and treatment. Our experience might give empirical information about the utility of two important diagnostic tools: the echocardiography, useful to accidentally identify the mediastinal mass and to follow up its consequences on cardiac haemodynamic, and the cardiac magnetic resonance, useful to provide information about the mass dimension and the contiguity relationship.

\section{Case Report}

We describe the case of a 29 -year-old woman at the 27th week of gestation that was admitted to the hospital for dyspnea, chest pain radiating to the left shoulder and fatigue. Physical examination was unremarkable except for an area of hypophonesis on the left basal lung compatible with pleural effusion and a tracheal murmur at the level of C7. An echocardiogram showed the presence of pericardial effusion $(\max 17 \mathrm{~mm})$ and revealed an echogenic mass, before the right ventricle, in the mediastinum with a disomogeneous echogenicity (Fig. 1a). In the suspicion of a mediastinal tumor, a chest $\mathrm{X}$ ray and subsequently a cardiac MRI were performed, this last better defined the mass dimension and its contiguity relationship and confirmed the suspicion of a lymphoproliferative disease (Fig. 1b). A biopsy was performed for the mass characterization and revealed an infiltrating non-Hodgkin's lymphoma at B big cells; chemotherapy was started. Following the mother willing and in accordance with a team of experts, it was planned to wait as much as possible for a safe caesarean delivery. Meanwhile the mother clinical and hemodynamic conditions and the fetus growth were monitored. Pericardial fluid and mass influences on cardiac hemodynamic were followed up by echocardiography until the 30th week of gestation when the instabilization of mother's clinical condition requested the interruption of pregnancy. The caesarean delivery succeeded excellently; the newborn was vital and normal for the gestation time. Through a transternal thoracothomy, after debriging of mediastinal, pulmonar, vascular and cardiac structures, the mass was excised.

The patient was discharged after ten days, up to today, after 6 months since surgery, results are quite satisfying; it 

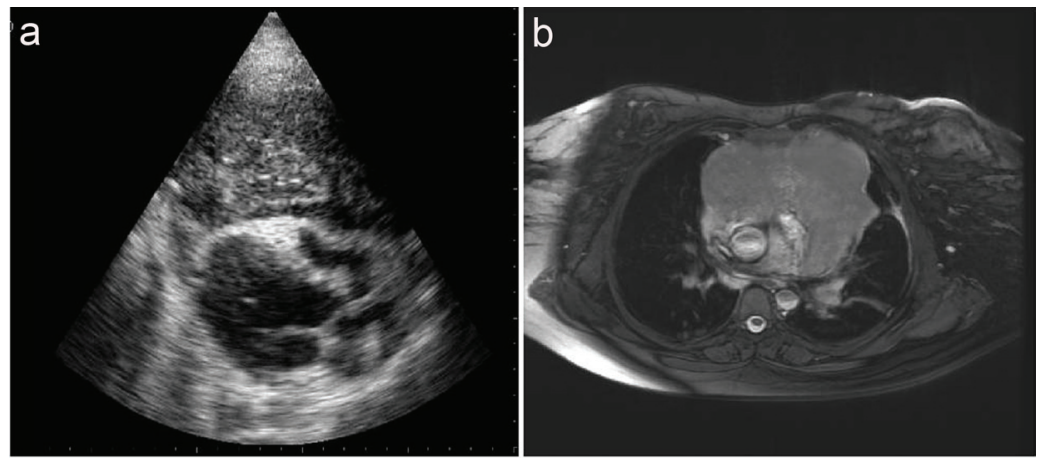

Figure 1. (a) Parasternal long axis view showing a disomogeneously echogenic mass, before the right ventricle, in the mediastinum. It modified the geometry of the right ventricle without impairing its filling. (b) Cardiac MRI image. It better defined the mass dimension and its contiguity relationship.

has been observed a reduction of mass dimension and a subjective wellness of the mother, the baby is doing very well.

\section{Discussion}

Non-Hodgkin's lymphoma during pregnancy is quite rare and often has a poor prognosis [1]. The tumor growth may be favoured by hormonal and immunitary changes typical of pregnancy. In most of cases they are low grade of differentiation lymphoma and they present mediastinal localization [2]. Among these, the primary mediastinal large B cell lymphoma, known as PMLBCL, is the rarest and its prognosis is controversial [3]. Diagnosis is often difficult because symptoms may be late and secondary to compression and infiltration of the nearer structures. The most frequent symptoms are dyspnea, cough, chest pain, compression of the superior vena cava, Corner syndrome, Pancoast syndrome, disphonia, palpitations, pleural effusion, and sometimes also cardiac tamponade [4].

There are no clear guidelines in the literature about the treatment of lymphoma inpregnancy. Some reports have documented the usefulness of biopsy for mass characterizationduring the gestation and others have documented the safety and efficacy of chemotherapy during pregnancy [1-6]. Echocardiography was a non-invasive, repeatable diagnostic tool able to identify the presence of the mediastinal mass and to follow up its consequences on cardiac hemodynamic.
Cardiac magnetic resonance was performed safety after the third trimester without producing apparent problems in the newborn. It helped for the diagnosis in giving information especially on the mass dimension and its contiguity relationship but did not characterize the mass.

\section{References}

1. Pereg D, Koren G, Lishner M. The treatment of Hodgkin's and non-Hodgkin's lymphoma in pregnancy. Haematologica 2007;92(9):1230-1237.

2. Pentheroudakis G, Pavlidis N. Cancer and pregnancy: poena magna, not anymore. Eur $\mathrm{J}$ Cancer 2006;42(2):126-140.

3. Maryniak RK, Roszkowska-Purska K. Primary mediastinal large B-cell lymphoma is an important differential among mediastinal tumours. Pol J Pathol 2002;53(3):139-144.

4. Silverman NA, Sabiston DC, Jr. Primary tumors and cysts of the mediastinum. Curr Probl Cancer 1977;2(5):1-55.

5. Morice P, Cristalli B, Heid M, Briere J, Levardon M. [Pregnancy and non-Hodgkin's lymphoma: a case]. J Gynecol Obstet Biol Reprod (Paris) 1993;22(1):68-70.

6. Nantel S, Parboosingh J, Poon MC. Treatment of an aggressive non-Hodgkin's lymphoma during pregnancy with MACOP-B chemotherapy. Med Pediatr Oncol 1990;18(2):143-145. 